\section{Eindimensionaler Ansatz überholt}

\author{
Um dem Konzept der nachhaltigen Entwicklung gerecht zu werden, muß sich \\ die immer noch naturwissenschaftlich-technisch dominierte Umweltforschung \\ nev ausrichten. Ökoforum, ein Kooperationsnetz von sieben Forschungsinstitu- \\ ten, fordert in dem folgenden Memorandum die Integration der Wirtschafts- \\ und Sozialwissenschaften und einen stärkeren Bezug zur Praxis.
}

$\mathrm{D}$

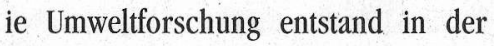
Bundesrepublik Deutschland vor rund 25 Jahren in enger Verbindung mit dem neuen Politikfeld Umweltpolitik. Sie stellt keine eigene Disziplin dar, sondern definiert sich umgekehrt über ihren Bezug zu spezifischen „Umweltproblemen“. Dieser Problembezug ist immer auch gesellschaftlich geprägt und explizit oder implizit - politisch beeinflußt. Lange Zeit war die Umweltforschung in der Bundesrepublik von einem politisch vorgegebenen Problembezug dominiert, der auf einem nachsorgenden und technikzentrierten Zugang sowie einem reduktionistischen Verständnis von ökologischen Problemen als „Störungen“ der natürlichen Umwelt beruhte.

Der Problembezug der. Umweltforschung hat sich in den letzten Jahren jedoch verändert verstärkt vor allem durch die Diskussion um das Konzept einer nachhaltigen und zukunftsfähigen Entwicklung (sustainable development). Dieses Konzept bedarf nicht nur einer theoretischen Klärung, wozu auch nach dem Stand der Debatte in der naturwissenschaftlichen Ökologie die Sozialwissenschaften beansprucht werden. Vielmehr lassen sich Umweltprobleme nicht mehr nur als Störungen und Überlastungen der natürlichen Umwelt begreifen, sondern auch als Folge unangepaßter gesellschaftlicher Handlungsmuster und Strukturzusammenhänge. Ent- sprechend stehen nun im Mittelpunkt weniger Untersuchungen von anthropogen gestörten natürlichen Systemen (,Ökosystemen“), sondern die dynamischen Wechselwirkungen zwischen gesellschaftlichen Handlungsmustern und natürlichen Wirkungsketten. Neben den Bezug auf natürliche Ressourcen und Systeme tritt somit ein Bezug auf gesellschaftliche Strukturen und Prozesse, z. B. auf Produktions- und Konsummuster. Lösungen können dann nicht mehr nur in neuen Techniken oder einem effizienteren Ökosystem-Mana- gement gesehen werden, sondern auch in Änderungen gesellschaftlicher Verhaltensmuster und Rahmenbedingungen.

.Vor diesem Hintergrund haben sich die Kriterien für eine vorsorgeorientierte, problem- und lösungsbezogene, disziplinenübergreifende Umweltforschung erweitert und präzisiert. Vor allem müssen nun die wirtschafts-, sozial- und kulturwissenschaftlichen Disziplinen gleichberechtigt neben den natur- und technikwissenschaftlichen in die Umweltforschung integriert werden (vgl. Gutachten des Wissenschaftsrats zur Umweltforschung in Deutschland). Darüber hinaus ist insbesondere die Einbeziehung von unterschiedlichen Akteuren und sozialen Gruppen mit ihren jeweiligen Handlungsmöglichkeiten als ein ganz entscheidendes Kriterium anzusehen. Allgemeiner formuliert kann von einer Öffnung der Umweltforschung hin zu einem verstärkten Handlungs- und Praxisbezug gesprochen werden. Deutlich ablesbar ist dies bei.spielsweise an neueren Förderkonzepten des Bundesforschungsministeriums (BMBF) wie „Agrarlandschaftsforschung“ oder „Nachhaltiges Wirtschaften“.

\section{Strukturdefizite der gegen- wärtigen Forschungslandschaft}

Umwelt und Ökologie sind seit den 70er Jahren in die bestehende Forschungslandschaft der Bundesrepublik eingefügt worden. Vor allem im Blick auf die skizzierten neuen Problemlagen und Ziele von Umweltforschung werden jedoch grundlegende strukturelle Defizite sichtbar:

- Nach wie vor ist die Umweltforschung naturwissenschaftlich-technisch dominiert (vgl. die Antwort der Bundesregierung auf die Anfrage der SPD-Fraktion von 1995). Theoretische, konzeptionelle, methodologische und organisatorische Voraussetzungen für die Durchfiuhrung problem- und lösungsorientierter interdisziplinärer Forschung von Natur- und Sozialwis- senschaften sind bislang so gut wie nicht entwickelt worden; sie sind als Innovation in der Forschungskultur inzwischen überfällig. Auch die Ökosystemforschung hat bisher aus institutionell-organisatorischen ebenso wie aus theoretischen und konzeptionellen Gründen eine interdisziplinäre Forschung in diesem Sinn nicht hervorgebracht.

- Noch immer überwiegen in der Umweltforschung der Bundesrepublik wissenschaftliche Großstrukturen (vor allem die Großforschungseinrichtungen). Dagegen fehlt es an Spielräumen für die Entwicklung neuer Arbeits- und Kooperationsformen (etwa flexible Verbünde oder Institute auf Zeit, wie sie der Wissenschaftsrat vorgeschlagen hat) und für neue organisatorische Strukturlösungen, die interdisziplinären, anwendungsbezogenen und ,vernetzten“ Forschungsansätzen genügen würden, etwa Institute oder Forschungszentren, an denen politik- und entscheidungsbezogene Forschung neben theoretischer und methodischer Reflexion möglich ist.

- Nach wie vor kann das Potential an den Hochschulen aus institutionellen wie aus Gründen eingefahrener Reproduktionsformen des akademisch anerkannten Wissens (Disziplinenorientierung, mangelnder Problem- und Gestaltungsbezug) nicht ausgeschöpft werden. Auch darauf hat der Wissenschaftsrat hingewiesen.

Selbst dort, wo durchaus Umorientierungen und Teilerfolge zu beobachten sind - etwa im Bereich der Förderkonzepte des BMBF oder der Neugründung von Forschungseinrichtungen werden die tradierten Strukturen letzlich doch nur punktuell modifiziert.

\section{Anforderungen an eine problemadäquate, innovative Umweltforschung}

Eine problemadäquate, gestaltungsorientierte Umweltpolitik ebenso wie die Konkretisierung und Umsetzung des Leitbilds einer nachhaltigen, zukunftsfähigen Entwicklung erfordern sowohl auf institutioneller als auch auf konzeptioneller Ebene einen neuen, innovativen Typus von Umweltforschung. Charakterisieren läßt sich dieser Forschungstyp durch die folgenden Kriterien:

- Problemorientierung mit Gesellschaftsbezug,

- Lösungsorientierung mit Akteursbezug,

- interdisziplinärer Ansatz unter Einschluß der Sozial-, Wirtschafts- und Kulturwissenschaften,

- Anwendungsorientierung bei gleichzeitiger 
Entwicklung neuer Methoden und Konzepte, flexible institutionelle Strukturen und hohe Kooperationsbereitschaft und -fähigkeit.

Dabei geht es nicht jedoch nur darum, wissenschaftlich-technische Lösungsvorschläge $\mathrm{zu}$ erarbeiten, sondern auch darum,

- eine Vermittlungsfunktion im Prozeß der gesellschaftlichen Entscheidungsfindung zu den wichtigen Fragen einer zukünftigen gesellschaftlicher Entwicklung wahrzunehmen;

- einen wechselseitigen Wissenstransfer zwischen den unterschiedlichen Akteuren zu ermöglichen und zu strukturieren, insbesondere zwischen den in der Regel voneinander abgeschotteten Bereichen von Wissenschaft, Politik und Öffentlichkeit einerseits sowie zwischen KonsumentInnen und Produzenten andererseits. Ein solcher Forschungstypus wird in den bisherigen Strukturen von Wissenschaft und Forschungspolitik nicht wirksam gefördert; er hat sich nur am Rande oder außerhalb der etablierten Forschungslandschaft in Ansätzen entwickeln können, etwa in den unabhängigen ökologisch orientierten Forschungsinstituten, die seit Ende der 70er Jahre entstanden sind. Gleichwohl hat dieser Forschungstyp in den letzten Jahren vor allem drei wichtige Funktionen für die Weiterentwicklung von Wissenschaft und Forschung insgesamt übernommen:

- Nachwuchsförderung und Ausbildungsarbeit für jüngere WissenschaftlerInnen;

- Entwicklung von „Prototypen“ für interdisziplinäre, problemorientierte Projektforschung; - innovative Impulse für die etablierten Sektoren der Forschungslandschaft.

\section{Resïmee}

Um eine Weiterentwicklung der Umweltforschungslandschaft hin zu einer ,nachhaltigen Forschungsentwicklung " zu gewährleisten, muß die Forschungspolitik ihr Instrumentarium in zahlreichen Bereichen umstrukturieren und an die skizzierten neuen Anforderungen und Zielsetzungen anpassen. Dazu gehört auch und vorrangig, bestehende innovative Potentiale, die überwiegend außerhalb der etablierten Forschungssektoren existieren, mit neuen Ansätzen $\mathrm{zu}$ fördern und zu stimulieren. Von besonderer
Bedeutung ist dabei die institutionelle Absicherung der entsprechenden Einrichtungen, die Verbesserung ihrer Kooperationsfähigkeit sowie die Sicherung der Qualität ihrer wissenschaftlichen Arbeit. Denn nur dann können von den bereits bestehenden innovativen Ansätzen auch weiterhin, und in stärkerem Maße als bisher, Veränderungsimpulse für die etablierten Sektoren der Forschungslandschaft ausgehen und dort die notwendigen Umstrukturierungen initiieren.

\section{Die Autorlnnen}

Im Ökoforum sind das Ökoinstitut Südtirol/Alto Adige, CONTRACT, das Institut für ökologische Wirlschaftsforschung, das Institui für sozial-ökologische Forschung, KATALYSE, das Öka-Inslitut e. V. und das Österreichische Ökologie-Instilut zusammengeschlossen.

Kontakt im Zusammenhang mit dem Memorandum u. a.: Thomas Jahn (ISOE),

Tel. 069/70 00 12; Heinz Kottmann (löW), Tel. $030 / 884594.0$

\section{... fïr denkende Anleger(innen) ...}

Sie wollen Ihr Geld rentabel und ökologisch sinnvoll anlegen?

Der Informationsdienst ÖKO-INVEST liefert Ihnen dazu alle zwei Wochen aktuelle und umfassende Informationen, z.B. über alternative Banken, aussichtsreiche "grüne“ Aktien oder ökologische Beteiligungsmöglichkeiten in Investmentfonds bzw. nicht börsennotierten Gesellschaften. Damit Sie wissen, welche Unternehmen sauber sind und es auch bleiben.

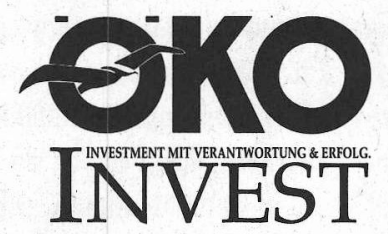

Ihr kostenloses Probeexemplar oder Infos zum Jahrbuch „Grünes Geld“ können Sie per Postkarte oder Fax abrufen bei der

\section{ÖKO-INVEST Verlags-GmbH}

Schweizertalstr. 8-10/5

A-1130 Wien

Tel. 0043/1/535 1815

Fax 0043/1/535 4669

\section{Effiziente Umwelt- und} Regionalplanung

- Profil: Dipl.-Volkswirt / Umweltauditor (TAE), 35 J., 5 Jahre Erfahrung im Projektmanagement städtebaul. Modellvorhaben und Mitwirkung an Umweltentlastungskonzepten.

Arbeitsschwerpunkte: Planungsökonomie, Wirtschaftlichkeitsanalyse und Standortmarketing für Erschließungsvorhaben mit ökol. Orientierung.

Ziel: Neues Betätigungsfeld im Bereich nachhaltiger Stadtentwicklungsplanung, Umweltökonomie und Umweltmanagement.

Zuschriften bitte an den ökom verlag z.Hd. Frau Huber Chiffre 112 
(c) 20I0 Authors; licensee IÖW and oekom verlag. This is an article distributed under the terms of the Creative Commons Attribution Non-Commercial No Derivates License (http://creativecommons.org/licenses/by-nc-nd/3.o/), which permits unrestricted use, distribution, and reproduction in any medium, provided the original work is properly cited. 